Voix et Images

voixetimages

\title{
Hosanna ou la quête d'une territorialité
}

\section{Roch Turbide}

Volume 7, numéro 2, hiver 1982

Michel Tremblay

URI : https://id.erudit.org/iderudit/200321ar

DOI : https://doi.org/10.7202/200321ar

Aller au sommaire du numéro

Éditeur(s)

Les Presses de l'Université du Québec

\section{ISSN}

0318-9201 (imprimé)

1705-933X (numérique)

Découvrir la revue

\section{Citer cet article}

Turbide, R. (1982). Hosanna ou la quête d'une territorialité. Voix et Images, 7(2), 307-318. https://doi.org/10.7202/200321ar d'utilisation que vous pouvez consulter en ligne.

https://apropos.erudit.org/fr/usagers/politique-dutilisation/ 


\title{
Hosanna ou la quête d'une territorialité
}

\author{
par Roch Turbide
}

Si la tâche du sémiologue est d'établir les systèmes de signes textuels pouvant permettre au metteur en scène et aux comédiens de construire un système signifiant où le spectateur concret trouve sa place, Hosanna est sans aucun doute la pièce de Tremblay qui accorde plus que tout autre un intérêt à cette tâche. Hosanna se présente en effet comme la pièce de Tremblay la plus piégée dans la mesure où tout semble ne fonctionner qu'à partir du moment où l'on hypostasie les personnages en personnes, qu'à partir du moment où le spectateur psychologise Hosanna comme un travesti aux prises avec des problèmes qui lui sont présentés. Hosanna rassure le critique théâtral traditionnel à la recherche d'une application encore possible de la psychologie, pratique qui nous paraît impossible puisque, comme le dit $A$. Ubersfeld, "ne saurait avoir d'inconscient ce qui n'a pas de psyché individuelle»'. Aussi, nous essaierons dans la présente étude de montrer comment, par le biais de l'espace dramatique ${ }^{2}$, il nous sera possible d'échapper à ce regard psychologisant pourtant impossible mais que seule la convention permet encore.

L'examen du modèle actantiel présenté par Hosanna ne nous permet pas à lui seul d'éviter ce piège précédemment dénoncé. Même si le modèle actantiel doit nous permettre de ne pas tomber dans les motivations psychologiques archaïques puisqu'il expose le désir qui propulse le héros dans le faire, celui que présente Hosanna s'avère toujours incomplet sans, comme nous le verrons plus tard, l'injection de ce que nous appelerons le "territoire". En effet, que veut Hosanna? Dès son entrée, tout désir semble éclaté, détruit. Ses premiers mots ne consistent qu'à dire: «J'voulais pus y aller. J'voulais pus... J'voulais pus y aller" ${ }^{3}$. L'imparfait et le passé composé, temps privilégiés par Hosanna, ne font qu'enchérir sur le passé de l'action d'Hosanna.

Moé, j'me disais... un jour, toué aussi tu vas faire ton entrée! Ah, ça va être une ben p'tite entrée à côté de ça, mais ça va être mieux que rien... Pis toutes les folles de Montréal vont chier du sang! (Silence) Ben mon entrée... dans Rome... (Silence) QUI C'EST QUI A CHIÉ DU SANG! QUI C'EST QUI A CHIE DU SANG! Cibole de marde ! ${ }^{4}$ 
Ses atouts dévoilés, sa défaite avouée, Hosanna ne voudra que raconter cet échec, comme pour mieux l'exposer afin de mieux l'assumer. Hosanna ne voudra même pas reprendre ce qu'elle ${ }^{5}$ a perdu, ce que l'on aurait pu voir comme action dynamique, puisque selon elle, rien n'est perdu : tout est plutôt changé.

Écoute ben ça, Cuirette! j'étais pu Cléopâtre, cibole, j'étais Samson! Oui, Samson! Pis j'ai toute démoli mes décors en papier mâché! Vous avez toutes démoli ma vie en papier mâché $!^{6}$

Ainsi, comment orienter ce désir d'Hosanna? Pierre Gobin énonce que "Cuirette, comme Hosanna, voudrait trouver la vérité, l'unité»7. Gobin aura donc dû identifier le désir d'Hosanna comme étant des «phantasmes a caractère à la fois obsessionnel et réifié" et affirmer que "son univers de rêve représente une recherche, une patience, une mise en scène ", que "Hosanna avait un seul rêve dans son univers" 8 . Bref, afin d'installer Hosanna dans un rôle de sujet, il nous faudrait tomber dans ce piège même que le repérage du modèle actantiel aurait dû nous éviter. Rien dans le texte ne vient représenter, ni même métonymiquement ou métaphoriquement, le supposé désir d'unité d'Hosanna. Au contraire, comme le dit Gobin, c'est l'échec cuisant du travesti (qui) révèle à Claude qu'il était fou ${ }^{9}$, donc entreprise d'Hosanna (purement psychologique) de prise de conscience et de décision de changement. II va sans dire que nous nous heurtons aux mêmes problèmes lorsque nous voulons attribuer le rôle du sujet au personnage de Cuirette. Si, comme le disait Gobin, Cuirette est lui aussi à la recherche de l'unité, nous devons suivre la même démarche que précédemment, faire face à ce même danger de psychologisation.

Même si elle n'est présente sur scène que par le truchement de la conversation téléphonique, par la parole d'Hosanna ou de Cuirette, Sandra pourrait prendre le rôle de sujet. Elle est, jusqu'à maintenant, la seule dont l'objet est clairement défini; Hosanna dit bien à Cuirette :

Essaye pas de me conter des histoires, ça poigne pas. Sandra, t'as jamais couché avec, pis tu coucheras jamais avec! (...) Elle, à voudrait, elle, a voudrait, mais pas toué. ${ }^{10}$

Ou encore, à Sandra au téléphone :

Aie, Sandra, Sandra! Cuirette est parti, y a une demi-heure... Y devrait ressoudre d'une minute à l'autre... T'as eu c'que tu voulais, ça fait qu'envoye, bande, grosse truie, bande!11

Le modèle actantiel devient alors évident: Sandra veut Cuirette pour satisfaire son propre désir sexuel, projet auquel s'oppose Hosanna. Jusqu'à maintenant, c'est le seul modèle actantiel possible bien qu'il soit encore contestable, les didascalies suivant cette réplique nous obligeant à remettre en question le rôle d'opposant attribué à Hosanna. Même si au dire de cette dernière, Sandra a eu ce qu'elle voulait, la chaîne événementielle montre, nous semble-t-il, le peu d'importance de cet épisode dans l'itinéraire que paraît suivre Hosanna, comme si le modèle actantiel déterminé 
ayant pour sujet Sandra n'était qu'un sous-modèle à un autre qui l'enchasserait, modèle principal qu'il serait impossible de déterminer de la façon employée jusqu'ici :

Elle raccroche brusquement.

Hosanna - Là, chus tannée!

Elle retourne à la coiffeuse, prend une chaise, vient l'installer dos au public, à l'avant-scène, puis s'assoit à cheval dessus. ${ }^{12}$

La présence de Reynald, “une nouvelle plotte»13, (même si cette présence ne se fait, comme dans le cas de Sandra, que par l'intermédiaire de l'appareil téléphonique) permet l'ébauche d'un autre modèle actantiel.

Mon Dieu, c'est rare que tu laisses

échapper une proie comme ça... ${ }^{14}$

Ainsi, nous avons la phrase «Cuirette veut satisfaire son propre désir sexuel, Reynald". Mais d'imputer à Hosanna le rôle d'opposant devient alors plus téméraire que lors du modèle actantiel ayant pour sujet Sandra. En effet, après que Cuirette eût laissé entendre qu'il partait, Hosanna dit bien qu'elle "aurait de besoin en cibole qu'(il) reste(s), par exemple» ${ }^{15}$, comme si Hosanna sentait qu'elle devrait s'opposer au projet de Cuirette, comme si son intuition lui disait que tel était son rôle; mais autre chose (impossible de déterminer jusqu'à maintenant) l'oblige à dire :

Mais j'veux ${ }^{16}$ pas que tu restes... ${ }^{17}$

La négation nie donc le rôle d'opposant d'Hosanna tout en interdisant l'attribution du rôle d'adjuvant puisqu'Hosanna, plutôt qu'aider, ne fait que refuser de s'opposer.

Toutefois, si on accepte que le personnage est un agrégat complexe groupé sous l'unité d'un nom ${ }^{18}$, on se rendra compte que le personnage global présenté dans les didascalies par le vocable «Hosanna est en réalité un tout composé de trois personnages possédant leur nom propre : 1) Hosanna, le travesti; 2) Liz Taylor dans Cléopâtre, dont les traces constituent en partie la représentation scénique du personnage global; 3) Claude, l'homme. Et, par une même logique, Cuirette se divise lui-même en deux personnages ; 1) Cuirette, I'homosexuel-motard déchu ; 2) Raymond, l'homme. La pièce Hosanna a donc plus de deux personnages scéniquement montrés : elle en a cinq qui, par le biais de la theâtralisation, se retrouvent réunis pour n'en former que deux.

Le problème rencontré quant à la détermination d'un verbe de volonté demeure évidemment toujours. Qu'Hosanna soit constituée de trois personnages ne change en rien l'absence de désir orienté. Mais il nous apparaît que le conflit se situe au cœur même de cette théâtralisation du personnage d'Hosanna, les trois personnages la constituant ne semblant jamais être présents simultanément sinon qu'en position conflictuelle. Ce simple fait nous permet de repérer un premier modèle déjà plus éclairant: Hosanna veut Cléopâtre. Sandra et les autres travestis présents au bar de 
Sandra prennent dans ce modèle un rôle d'opposant (inconditionnel) à Hosanna alors que Cuirette, personnage ambigu de par ces deux rôles, aide inconditionnellement Hosanna mais, dans un même temps, se fait prendre au piège tendu par Sandra, aide cette dernière et devient alors opposant au projet d'Hosanna.

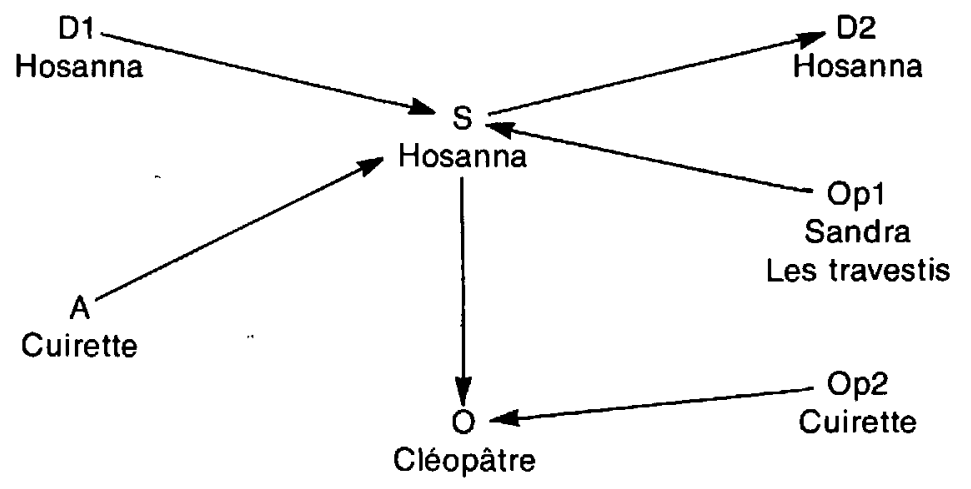

II faut ici spécifier que bien qu'elles soient pour le moment suffisantes, les dénominations du destinateur D1 et du destinataire D2 sont très incomplètes. II faudrait ajouter à la liste des destinateurs les valeurs esthétiques américaines qui font d'Élizabeth Taylor un idéal esthétique, les interprétations actuelles du personnage historique qu'est Cléopâtre, etc. Nous arriverions peut-être alors aux conclusions qu'Hosanna est plus américaine que québécoise, ou encore, si nous extrapolons, que la société québécoise est culturellement américaine de par ses choix d'objets de désir. De même, il faut se demander: pour qui Hosanna veut-elle Cléopâtre? Pour elle-même et nulle autre qu'elle-même n'en profitera. Nous pouvons dès lors voir comment nous pourrions affirmer que l'action d'Hosanna est asociale, ahistorique, que son action est non pas un rapprochement, mais un éloignement de la société, de l'histoire, du monde, donc entreprise purement aliénante parce que figée sur une seule image, soit celle de Cléopâtre.

Ce modèle ne rend compte que de la situation initiale, encore est-il qu'il nous paraît dès le départ périmé, Hosanna ne faisant que constater l'impossibilité d'un tel modèle. Aussi, sera-t-il plus juste de renverser ce premier code. Nous aurons alors : 


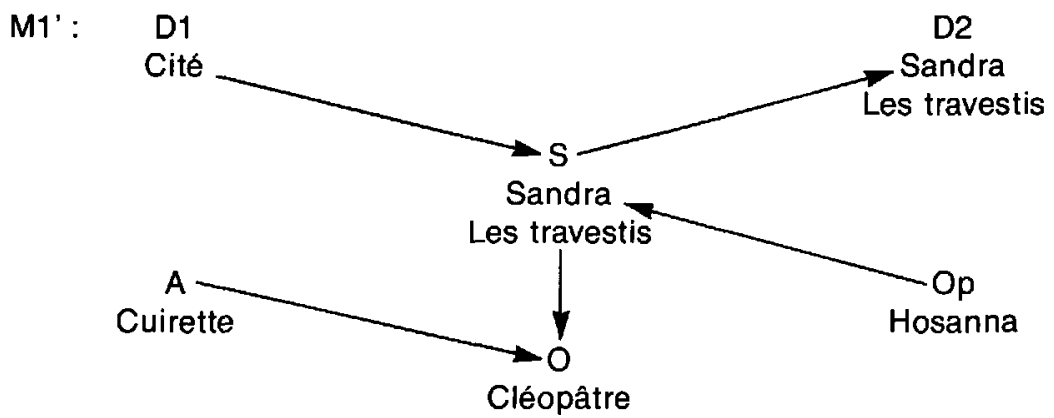

Nous employons ici le terme "Cité" comme D1 dans la mesure où dans cette société de travestis, l'obtention de l'objet Cléopâtre semble correspondre à une certaine reconnaissance sociale, voire à une position de pouvoir.

Par ailleurs, la situation d'arrivée met en place un autre modèle. En effet, la pièce se termine quand Hosanna «se lève lentement, enlève son slip et se retourne, nue, vers Cuirette" pour lui dire

R'garde, Raymond, chus t'un homme! Chus t'un homme, Raymond! Chus t'un homme! Chus t'un homme! Chus t'un homme!,

pour ensuite voir Cuirette-Raymond "se leve(r), se dirige( $r$ ) vers Claude et le prend(re) dans ses bras"19. Ainsi, la fin de la pièce dépendrait de deux objets que doit obtenir Hosanna : cet état d'homme qu'elle semble retrouver et que l'on a dénommé 'Claude' et le retour dans les bras de Cuirette:

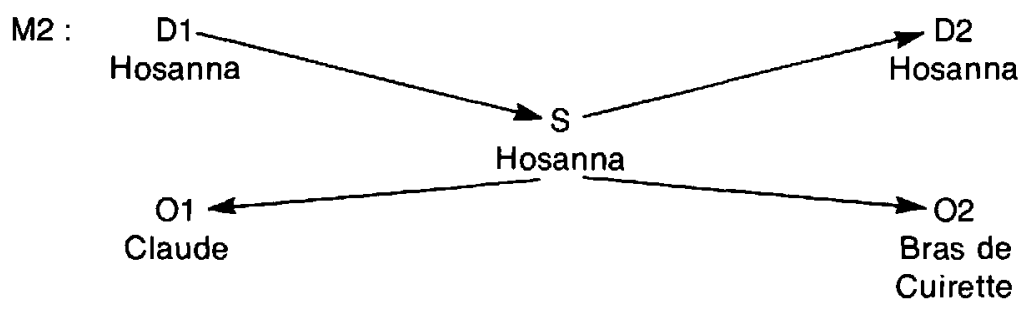


Toutefois, une question demeure : comment se fait le passage du modèle M1' au modèle M2 ? Rien ne vient textuellement expliquer la nécessité de ce dernier modèle, ni même l'annoncer. Or, c'est par la compréhension du fonctionnement du système spatial que nous comprendrons ce passage, l'espace devenant ici la raison même de l'existence du modèle M2.

Auparavant, il nous faudra y aller de quelques considérations théoriques. Nous ne reprendrons pas ici tous les rôles que peut jouer l'espace, qu'il soit dramatique ou scénique ${ }^{20}$. Nous ne reprendrons ici que l'idée d'Anne Ubersfeld qui montre comment l'on peut déterminer dans un texte dramatique donné deux ensembles paradigmatiques qui en principe n'ont pas d'intersection (au sens mathématique du terme). Elle donne alors l'exemple du roi qui est roi dans son espace mais qui est non-roi dans un autre espace 21 .

A partir de cet exemple, il sera facile de comprendre ce que nous définirons comme le concept de «territoire». Au risque de nous répéter, le personnage n'a pas d'âme. Il est un être de papier, un "corps sans organe " 22 dont l'identité est révélée au spectateur par le type de discours dont il est l'énonciateur, par le rôle actantiel qu'il joue au sein de l'action, etc. Or, l'espace peut lui aussi jouer le rôle d'interprétant (dans le sens piercien du terme) du signe qu'est le personnage ${ }^{23}$. Si le roi est roi dans son espace et qu'il est non-roi dans un autre espace, c'est que, par un jeu de réversibilité, l'espace (qu'il soit figuré ou non, figurable ou non) définit le personnage ou, plus encore, en est l'identité. Le "territoire" est donc cet aspect de l'espace (contenant-contenu) qui, en indiquant le rapport du personnage au monde, permet au personnage d'être ce qu'il est. Aussi, c'est non seulement parce que Hosanna tient un discours de travesti qu'elle en est un. C'est aussi parce qu'elle vit dans un espace de travesti, espace donné à construire par le texte et que la représentation a l'avantage de donner à voir ${ }^{24}$.

Si nous revenons au texte Hosanna, nous pouvons dire que Hosanna, autant que Cuirette, ont parcouru dans le sens propre du mot un certain itinéraire. Hosanna est partie de Saint-Eustache pour arriver à Montreal alors que Cuirette a fait ses débuts dans le parc Lafontaine pour arriver au véritable monde d'Hosanna et que nous conceptualiserons par le terme "territoire Montréal». Ce territoire Montréal inclut évidemment tous les lieux géographiques référentiels tels la Plaza Saint-Hubert, la rue Bélanger, la rue Beaubien, le théâtre Scala, la Place Ville-Marie, les " meat-racks ", etc., lieux que met en place le texte. De même, ce territoire comprendra les lieux narratifs fictionnels (lieux qui permettent l'implantation de la trame événementielle) ${ }^{25}$ que sont l'appartement d'Hosanna, le club chez Sandra, la maison de Sandra, etc.

Par ailleurs, il est possible de voir comment Hosanna s'est créé un autre territoire: Rome. En examinant la narration que fait Hosanna dans son monologue du deuxième acte, nous nous rendons compte qu'avant de se 
rendre chez Sandra, Hosanna n'était plus seulement Hosanna mais un nouveau personnage Hosanna-Cléopâtre.

Hosanna était enfin prête à faire son entrée dans Rome! ${ }^{26}$

Une telle réplique montre comment Hosanna avait réussi à concrétiser un deuxième territoire, ou plus encore, à fusionner le territoire Rome et le territoire Montréal dans lequel elle se mouvait. Hosanna affirme clairement son travail sur son propre territoire, donc sur son identité :

Trois semaines d'excitations, trois semaines à me garrocher partout, d'un bord pis de l'autre, pour essayer de rapailler les bebelles, les crémages à gâteaux, les matériels ridicules (...). ${ }^{27}$

En parlant de sa robe (contenu du territoire Rome), elle affirme sa réussite :

(...) j'me disais qu'était encore plus belle que dans mes rêves ! ${ }^{28}$

Nous voyons comment, par le rapprochement de leurs objets respectifs, Hosanna a réussi à ne faire de ces deux territoires qu'un seul. Nous pouvons donc dire qu'au début de la pièce, les personnages Cuirette et Hosanna ont chacun leur propre univers composé de différents territoires dont un qu'ils partagent.

Presqu'au début de la pièce, Hosanna spatialise clairement son conflit avec Sandra et avec, parce qu'ils sont tous un seul et même acteur ${ }^{29}$, les travestis: à la suite d'une remarque de Cuirette qui tente d'excuser Hosanna auprès de Sandra, Hosanna s'empresse de répondre:

Fais toé z'en pas, ça fait quatre ans qu'à veut se mettre à ma place, la chienne! ${ }^{30}$

Cette réplique constitue un premier indice du réel conflit : Hosanna occupe un territoire Montréal-Rome que veulent lui enlever Sandra et les travestis. On comprend alors tout le drame du club chez Sandra. Tous les travestis, en s'habillant en Cléopâtre, se sont accaparé des objets contenus ${ }^{31}$ dans le monde de Cléopâtre et l'en ont, par conséquent, dépossédée. En examinant les agissements d'Hosanna envers les objets qui l'entourent, on se rend compte que celle-ci, comme si elle avait compris qu'ils lui avaient été volés, qu'ils avaient été vidés de leur sens, est amenée à entreprendre le travail du deuil au sujet de ces objets (en termes freudiens, nous pourrions dire qu'il s'agit de délibidinaliser ces objets). Mais dans un même temps, nous voyons les écueils d'une telie vision : comment manifester théâtralement cette évolution que nous sommes plutôt tenté de voir comme cheminement psychologique alors que, nous l'avons déjà dit, le theâtre est un monde d'où la psychologie, de par la notion de personnage, est expulsée?

Nous sommes, à première vue, porté à croire que ce que donne Tremblay comme lieu scénique est espace unique : l'appartement d'Hosanna. Or, dans le deuxième acte, Hosanna, sans sortir de la scène, quitte l'appartement en tant que tel pour venir à l'avant-scène, lieu vide par excellence, où elle apporte la chaise de la coiffeuse : 
Elle retourne à la coiffeuse, prend une chaise, vient l'installer dos au public, à l'avant-scène, puis s'assoit à cheval dessus. ${ }^{32}$

C'est donc par la mise en place de l'avant-scène d'où Hosanna choisit le public comme confident que Tremblay traduit le plus théatralement possible la trame d'Hosanna: Hosanna fut expropriée de son territoire et, puisque c'est ce dernier qui la définissait (même plus, lui conférait son existence), elle se voit contrainte de sortir définitivement de ce territoire, de se mettre littéralement à nu dans un territoire lui-même nu puisque vide de tout objet.

C'est ainsi que s'expliquent les mouvements scéniques d'Hosanna qui retourne, lors de sa narration à partir de l'avant-scène, sur la scène en tant que telle. En effet, après qu'elle eût raconté une partie des événements qui s'étaient produits chez Sandra, «Hosanna ouvre les yeux, se lève et se dirige vers la fenêtre qu'elle ouvre " ${ }^{33}$, comme si elle étouffait dans ce lieu vide de l'avant-scène, demande une cigarette; puis, "elle referme la fenêtre et revient s'asseoir " ${ }^{34}$ pour reprendre sa narration. Elle retourne ensuite dans son territoire initial pour y chercher dans le placard la robe de Cléopâtre qui, par un recreusement symbolique, représente ce rapport au monde de travesti-Cléopâtre qu'elle remet alors en question. Elle exprime de la façon la plus brutale son rejet de ce monde: "Elle lance la robe par terre „35. Par la suite, «elle prend sa robe dans ses bras et se rassoit " 36 pour renouer avec le passé et reprendre le fil de sa narration. Elle retournera plus tard dans ce territoire auquel elle tente d'échapper: "Elle dépose la robe, prend la chaise et va s'installer à sa table de maquillage " 36 . Mais ce sera encore une fois pour briser un autre de ces liens que les objets représentaient : “Elle prend doucement un pot de fond de teint. (...) Elle lance le pot par terre et se redresse ${ }^{37}$. Après avoir rejeté la robe de Cléopâtre qui signifiait le plus son occupation du territoire Rome, Hosanna éloigne et détruit le pot de fond de teint qui symbolisait peut-être le mieux son rapport au monde de travestis. Finalement, Hosanna retourne à l'avantscène et y reste jusqu'à l'arrivée de Cuirette.

Evidemment, Hosanna n'est pas la seule à être privée de ses rapports au monde: Cuirette est lui aussi obligé de repenser sa relation au monde. Il a débuté sa carrière, comme il le dit lui-même, au parc Lafontaine :

J'me suis dit: Cuirette, mon gars, y'ont construit un théâtre pardessus la place ousque t'as faite tes débuts... ${ }^{38}$

Mais Cuirette nous annonce par la suite comment son territoire fut à lui aussi volé :

Des lumières, ciboire! Partout! Y'ont toute illuminé mon parc Lafontaine! J'ai pété deux lampadaires, mais c'était des vieux, pis y sont beaux, ça fait que j'ai arrêté. On peut même pas péter les nouveaux lampadaires, sont trop hauts! $Y$ fait aussi clair que sur le terrain de baseball. Partout ${ }^{39}$ 
Toutefois, contrairement à Hosanna, Cuirette, même s'il spécifie qu'il déteste le changement, assume le $\mathrm{v}(\mathrm{i})$ ol de son territoire en affirmant que :

J'aime pas que les affaires changent. J'criais: Gang d'hosties, d'écœurants, vous avez tout changé! Maudits chiens sales! Vous avez peur qui reste des coins noirs, hein, vous mettez des lumières partout! Ben sacrement, on va vous faire ça dans' face. (...) Les hosties! Y'ont toute changé le parc Lafontaine, Hosanna! Même ça!40

Nous pouvons voir comment le passage du modèle M1' au modèle M2 s'explique clairement puisque l'inter-relation des actants sera toujours modulée par la notion de territoire. Les sujets Sandra et les travestis veulent le territoire de Cléopâtre dans lequel vit Hosanna, l'obtiennent et, consequemment, en expulsent Hosanna qui doit alors rechercher un deuxième territoire, territoire qu'elle ne pourra habiter que dans son aspect Claude. Cuirette, de son côté, incapable de se recréer un territoire, ne pouvant qu'en assumer le vol (d'où sa faiblesse apparente) accepte alors de se plier à la volonté d'Hosanna qui l'inclut dans son propre territoire à creer, Cuirette étant la seule composante du territoire d'Hosanna dont Sandra et ses acolytes n'ont pas réussi à s'accaparer. C'est ainsi que prend son véritable sens le rapprochement que fait Hosanna dans son avant-dernière réplique :

Cléopâtre est morte, pis le Parc Lafontaine est toute illuminé !41

Tout est maintenant résolu, Hosanna déclare fermée la boucle de l'intrigue : l'objet de désir qu'est Cléopâtre n'existe plus et on a assumé le vol de son territoire.

Par ailleurs, en plus de fournir les éléments pouvant servir à l'établissement des paradigmes auxquels appartiennent les personnages (puisqu'ils seront définis par les éléments du territoire qu'ils habitent), en plus de spatialiser non seulement le conflit mais les enjeux des systèmes que sont la temporalite et le discours ${ }^{42}$, la spatialité, on s'en rendra compte, a avec le discours dans un rapport constant et réversible, qu'elle a constamment commentaire et sujet ${ }^{43}$ l'un de l'autre. Un simple remarque d'Hosanna nous permet de voir comment le discours s'accapare de la spatialité qui, tout en en étant l'objet, détermine le discours :

Écoute ben ça, Cuirette ; j'étais pus Cléopâtre, cibole, j'étais Samson! Pis j'ai toute démoli mes décors en papier mâché! Vous avez toutes démoli ma vie en papier mâché! (Pause) J'savais pas que vous m'haissiez tant que ça... ${ }^{44}$

L'espace et le temps sont aussi dans un même rapport, l'espace servant à exprimer de la façon la plus concise la confusion des passés rapportés:

Hosanna faisait son entrée! Hosanna, assise dans son chariot avec des curieux après le banc pour pas qu'y balance, déguisée comme Elizabeth Taylor, avec dans les mains un fouet pis une boule en or en papier mâché; Hosanna faisait son entree dans Rome! Pis pendant ce temps-là, l'enseigne de la Pharmacie Beaubien garrochait ses lumiè- 
res rouges pis jaunes dans ce miroir, pis Cuirette ronflait... (Silence) L'enseigne de la Pharmacie Beaubien garrochait ses lumières rouges pis jaunes dans le miroir, pis Cuirette ronflait... mon bras autour du cou d'Hosanna qui faisait son entrée triomphale dans Rome $!^{45}$

Évidemment, oublier qu'une représentation peut faire éclater une pièce en mille éclats, assumer que le metteur en scène construit à chaque fois un présupposé identique à celui qu'avait injecté l'auteur dans son texte écrit, serait faire preuve d'une naïveté manifeste. De même, dire que notre analyse nous permet de découvrir le sens d'Hosanna nous apparaîtrait comme un réel abus de pouvoir. Dire qu'elle en a dévoilé les sens nous paraît encore dangereux. Peut-être pouvons-nous dire qu'elle a permis de découvrir la cohérence d'Hosanna, nous a permis d'y déconstruire l'apparente anarchie du message, d'y relever les virtuelles conditions de production de sens à partir du discours textuellement livré par Tremblay.

Par ailleurs, d'un ordre purement théâtrologique, nous savons que la représentation peut modifier totalement la base même de notre étude, c'est-à-dire le texte. Il nous suffit de donner l'exemple du clown qui ne fait de la parole (que résume le texte) qu'un complément d'une gestualité qu'elle ponctue, pour souligner la fragilité de la permanence textuelle. La mise en scène de la pièce qui ferait du discours d'Hosanna une pure et simple confession (et ce, en transgressant les mouvements scéniques que demande Tremblay dans la couche didascalique) suffirait à enlever aux lieux la possibilité de traduire cette réalité que notre étude a tenté de dévoiler; elle suffirait à détruire la compréhension d'Hosanna comme l'itinéraire d'un prédicat qui se véhicule à travers des figures territoriales. On ferait alors exploser la parole par une gestualité qui n'a aucun rapport avec elle. Ou encore, qu'est-ce qui empêcherait une mise en scène d'aplatir cette parole en installant tout simplement une situation scéniquement physique qui ferait exactement comme s'il n'y avait pas moyen que les mots disent autre chose que ce qu'ils disent littéralement? L'enjeu de la pièce que nous a dévoilé notre étude pourra alors toujours servir d'étalon de base pour mesurer l'écart que se sont permis en tant que scripteur le metteur en scène ou les autres comédiens, pour voir quels outils de production de sens, ces scripteurs ont utilisés et dont il nous faudra évaluer la pertinence, le rôle, la signifiance.

1. UBERSFELD, Anne. Lire le theatre. Paris, Ed. sociales, Coll. "Les classiques du peuple critique $m, 1978$, p. 139.

2. L'espace dramatique est l'aspect de l'espace theatral faisant de ce dernier un lieu à construire. C'est l'espace suggéré par le texte, autant par ses didascalies que ses dialogues.

3. TREMBLAY, Michel. Hosanna, Montréal, Leméac, Coll. Répertoire québécois, 1973 , p. 12.

4. Ibid., p. 18.

5. Pour éviter toute confusion, nous avons, par convention, décidé d'utiliser ici le pronom coréférentiel féminin et ce, à l'endroit de tous les travestis. 
6. TREMBLAY, Michel. Op. cit., p. 74.

7. GOBIN, Pierre. Le fou et ses doubles. Montréal, P.U.M., Coll. Lignes québécoises, 1978, p. 224.

8. Ibid., p. 224.

9. Ibid., p. 225.

10. TREMBLAY, Michel. Op. cit., p. 26.

11. Ibid., p. 17.

12. Ibid., p. 17.

12. Ibid., p. 37.

13. Ibid., p. 34.

14. Ibid., p. 74

15. Ibid., p. 34.

16. En italique dans le texte de Leméac.

17. TREMBLAY, Michel. Op. cit., p. 34.

18. Nous faisons ici référence aux propositions de Madame Ubersfeld dans son essai Lire le theâtre, p. 119-151.

19. TREMBLAY, Michel. Op. cit., p. 75.

20. Nous préférons vous renvoyer au chapitre de Lire le théatre traitant de ce sujet.

21. UBERSFELD, Anne. Op. cit., p. 187-188.

22. Pour reprendre l'expression de Deleuze et Guattari.

23. En lui donnant le rôle d'interprétant, il est évident que nous considérons cet espace comme composante même du signe-personnage, cet espace étant un élément tout aussi essentiel à la relation triadique du signe que la représentation et l'objet.

24. Il serait d'ailleurs presque impossible d'imaginer Hosanna dans un autre espace que celui-là; cet autre décor serait alors un signe parasitaire absolu dans la mesure où il déplacerait le réel enjeu de la pièce, transformerait Hosanna en une dernière Madame Bovary puisque son discours serait en total désaccord avec son rapport au monde.

25. Les lieux narratifs fictionnels ne sont présents que pour permettre l'implantation de la trame événementielle alors que les lieux référentiels, tout en jouant le rôle des lieux fictionnels, renvoient à une réalité concrète et extérieure et ont, par conséquent, un rôle additionnel qu'est la fabrication d'un certain pittoresque.

26. TREMBLAY, Michel. Op. cit., p. 62.

27. Ibid., p. 61.

28. Ibid., p. 62.

29. Entendu dans le sens de particularisation d'un actant.

30. TREMBLAY, Michel. Op. cit., p. 19.

31. Plus précisément, du vêtement-contenu qui est en même temps l'origine et la définition (d'où son rôle symbolique) de la Rome hollywoodienne et montréalaise d'Hosanna.

32. TREMBLAY, Michel. Op. cit., p. 57. Il est intéressant de constater que même la chaise perd son statut initial de "chaise de coiffeuse" puisque Hosanna la place dos au public pour s'asseoir d'une façon quelque peu particulière.

33. TREMBLAY, Michel. Op. cit., p. 60.

34. Ibid., p. 60 .

35. Ibid., p. 61.

36. Ibid., p. 63.

37. Ibid., p. 64

38. Ibid., p. 16.

39. Ibid, p. 16

40. Ibid., p. 69.

41. Ibid., p. 75.

42. Une analyse du discours d'Hosanna révèle en effet le réel problème de celle-ci : il consiste en une inadéquation du «je " d'Hosanna et du discours dont elle est l'énonciatrice, et ce, dans un monde où la parole constitue la marchan- 
dise de base, la monnaie d'échange. De même, le territoire "nu" correspond à la situation temporelle d'Hosanna qui s'avère incapable de faire le saut dans le futur que présuppose habituellement la fin d'une pièce.

43. Commentaires et sujet sont employés ici comme traduction des termes "topic" et "comment" qu'utilise la philosophie du langage.

44. TREMBLAY, Michel. Op. cit., p. 74.

45. Ibid., p. 60. 